\title{
New observations of the Geminga pulsar at low radio frequencies
}

\author{
V. M. Malofeev, O. I. Malov, S. V. Logvinenko and D. A. Teplykh \\ Pushchino Radio Astronomy Observatory, \\ 142290, Pushchino, Moscow reg., RUSSIA \\ email: malofeev@prao.ru
}

\begin{abstract}
New evidence for the detection of Geminga at three low frequencies is presented. The observations were carried out on two sensitive transit radio telescopes in the range 42-112 MHz. We used three new digital receivers to detect the pulses and to obtain dynamic spectra. The exact value of the dispersion measure has been calculated.
\end{abstract}

Keywords. Pulsars.

\section{Introduction}

The famous neutron star Geminga was the first astronomical object discovered through its $\gamma$-ray emission, in 1975 (Kniffen et al. 1975). The detection of coherent pulsation with a period of $237 \mathrm{~ms}$ in X-ray emission and the pulsed $\gamma$ - radiation were next reported in 1992 (Halpern \& Holt 1992, Bertsch et al. 1992). Three groups reported the detection of pulsed radio emission from Geminga at frequency $102.5 \mathrm{MHz}$ (Malofeev \& Malov 1997; Kuzmin \& Losovsky 1997; Shitov \& Pugachev 1998). One group confirmed the pulsed radio emission at the same frequency of $103 \mathrm{MHz}$ (Vats et al. 1999). Recently weak continuum radio emission has been detected at the frequency $4.8 \mathrm{GHz}$ (Pellizzoni et al. 2011).

\section{Observations and results}

The observations were carried out on two sensitive transit radio telescopes in Pushchino in the range $42-112 \mathrm{MHz}$. They have been made at $111.5 \mathrm{MHz}$ using Large Phase Array (LPA) antenna, with is a transit phased array comprising 16384 dipoles and covering an area of 18 acres. Its operating frequency is $111.5 \pm 1.5 \mathrm{MHz}$ and the telescope is a sensitive instrument with an effective area of $\simeq 3 \cdot 10^{4} \mathrm{~m}^{2}$. The high-sensitivity DKR-1000 (EastWest arm), which operates at $30-110 \mathrm{MHz}$, has an effective area of $\simeq 7000 \mathrm{~m}^{2}$ and an observing session duration of $15^{\mathrm{m}} / \cos \delta$. New series of observations were obtained using a unique set of digital, multi-channel receivers designed for pulsar observations, which came into use in 2006-09. The width of the operational frequency band is $2.5 \mathrm{MHz}$, which is separated by the FFT into 512 spectral channels with widths of $4.88 \mathrm{kHz}$ each. The reduction programmes implement several techniques for removing interference, using several criteria for distinguishing false pulses from real signals (for details see Malofeev et al. 2012).

In the beginning of this year new observations in Pushchino showed the evidence for the detection of Geminga at three low frequencies during two months (JanuaryFebruary of 2012) in a few sets of observations. Here, we present the results for three days 19 - 21 of January. To check for the presence of the weak pulsar signal and to raise the reliability, we determine the observing window or the group as three apparent pulsar 


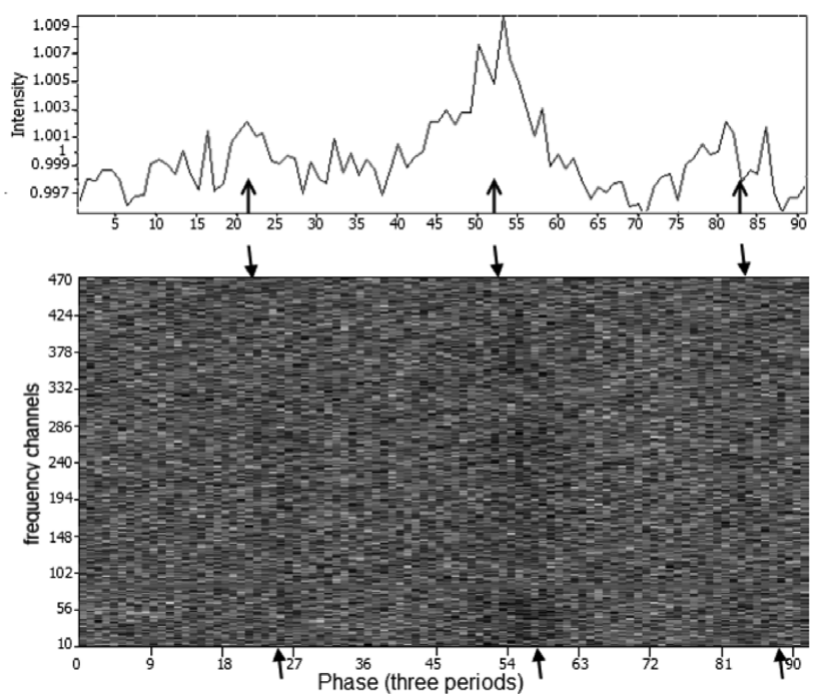

Figure 1. Example of a pulse profile (upper) and a dynamical spectrum (lower) of Geminga at $111 \mathrm{MHz}$, obtained by summing 36 selected groups (triple periods) on the 20.01.12. The horizontal axis is in samples of the triple period of the pulsar. The dispersion track is marked by arrows.

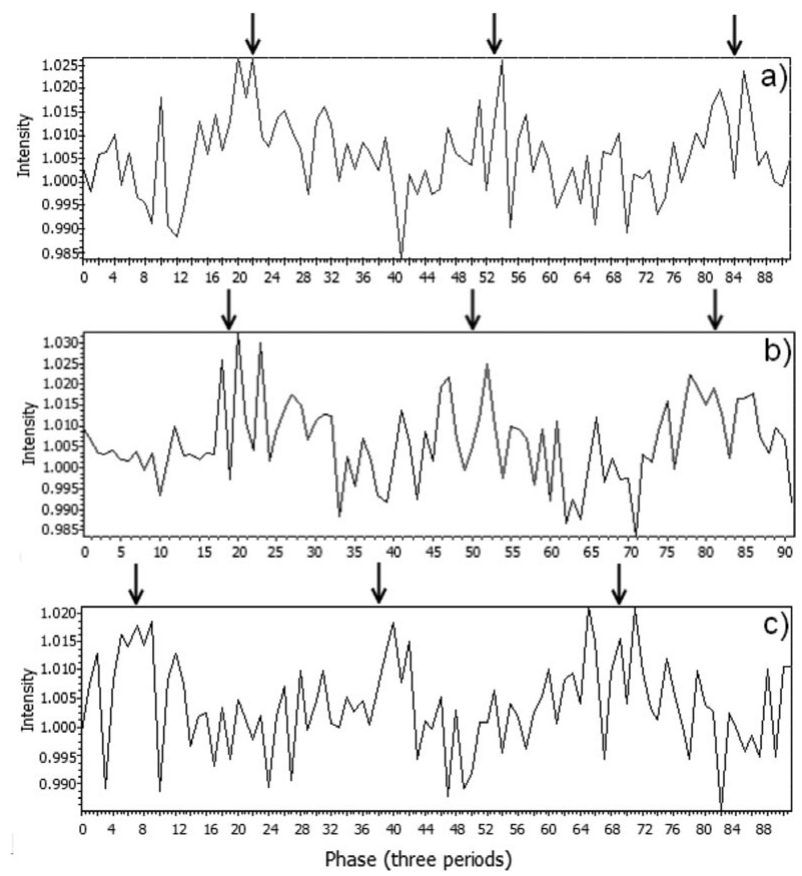

Figure 2. Examples of an individual pulse profiles of Geminga at $111 \mathrm{MHz}$ on the 20.01.12. The horizontal axis is in samples of the triple period of the pulsar. The phases of pulses are marked by arrows.

period with the sampling interval $7.5776 \mathrm{~ms}$. One observation set contained 280 or 841 groups (triple periods) at frequencies $111 \mathrm{MHz}$ and 42/62 $\mathrm{MHz}$ accordingly. The direct integration of all groups showed week signal with signal-to-noise $(\mathrm{S} / \mathrm{N})$ ratio about 5 in some observations. But if we used the method of visible pulses selection (Malofeev et al. 


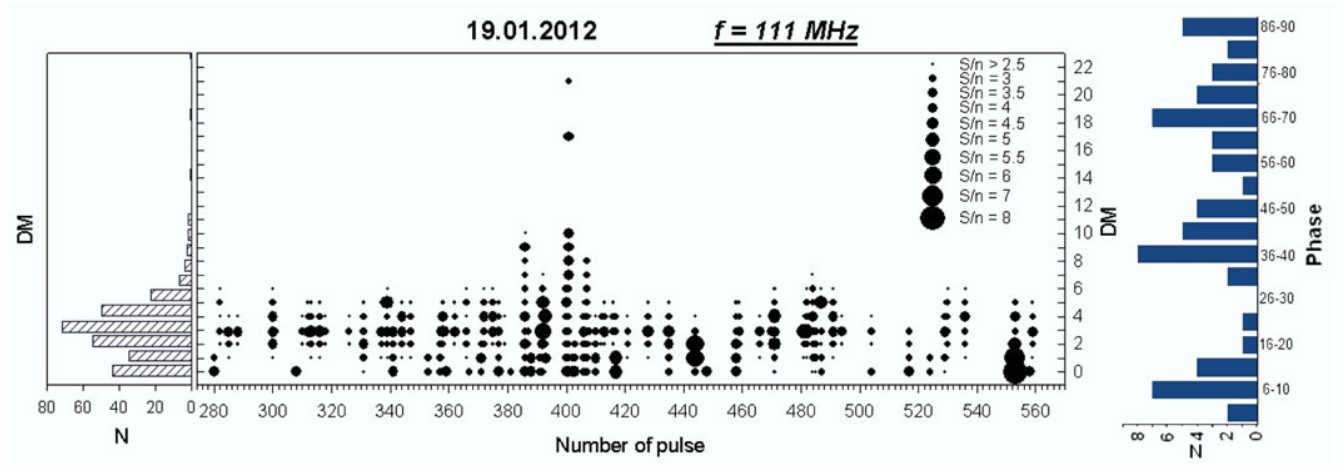

Figure 3. The central panel shows events with $S / N>2.5$ at different DM versus number at pulse (time). DM with larger circles denoting stronger signal. The left panel shows the histogram of the number of events versus DM. The right panel shows histogram of the events with $S / N>2.5$ versus of period phase for 19.01.2012 (triple period).

2012 ) for reduction of data in these observations, the value of $\mathrm{S} / \mathrm{N}$ ratio can reach more than 10 in these days (Fig. 1). In this case we summed all groups, where have been the pulses with $S / N>2$ at selected phase. The mean profile of 36 such selected groups is presented at Fig. 1 (upper). The integration was carried out at phase sample $52 \pm 3$, but possible see two other more week pulses at the phases near samples 21 and 83 . All three pulses are separated by one pulse period (31.29 samples). Next very important thing is the presence of the signal dispersion. The dispersion tracks are seen at dynamic spectra (Fig. 1, bottom panels). We have been luck and first time new simultaneous observation at three frequencies on 20 of January give us possibility to measure more exactly the value of $D M=2.89 \pm 0.02$. Fig. 3 shows events with $S / N>2.5$ versus number of pulse, $\mathrm{DM}$ and phase of period for 19.01.2012 at $111 \mathrm{MHz}$

\section{Acknowledgements}

This work was financially supported by the Russian Foundation for Basic Research (project code 12-02-00661) and Academy of Science (programme nonstationary phenomenon in the objects of the Universe, 2012).

\section{References}

Kniffen, D. A., et al. 1975, Proc. 14th Int. Cosmik Ray Conf., 1, 100

Halpern, J. P. \& Holt, S. S. 2005, Nature, 357, 222

Bertsch, D. L., et al. 1992, Nature, 357, 306

Malofeev, V. M. \& Malov, O. I. 1997, Nature, 389, 697

Kuzmin, A. D. \& Losovsky, B. Y. a. 1997, Pis'ma AZh, 23, 323

Shitov, Yu. P. \& Pugachev, V. D. 1998, New Astronomy, 3, 101

Vats, H. O., Singal, A. K., Deshpande, M. R., et al. 1999, MNRAS, 302, 65

Pellizzoni, A., et al. 2011, MNRAS, 416, 45

Malofeev, V. M., Teplykh, D. A., \& Logvinenko, S. V. 2012, Astronomy Reports 56, 35 